\title{
a dimensão política do processo de formação de pessoal AUXILIAR: A ENFERMAGEM RUMO AO SUS ${ }^{1}$
}

Alva Helena de Almeida ${ }^{2}$ Cássia Baldini Soares ${ }^{3}$

Almeida AH, Soares CB. A dimensão política do processo de formação de pessoal auxiliar: a enfermagem rumo ao SUS. Rev Latino-am Enfermagem 2002 setembro-outubro; 10(5):629-36.

A mudança de administração no município de São Paulo em 1989 inovou a gestão dos serviços de saúde, objetivando a implementação do Sistema Único de Saúde (SUS) e tendo como uma estratégia fundamental a qualificação dos trabalhadores. Este artigo objetiva analisar a incorporação dos princípios do SUS aos cursos de formação de auxiliares de enfermagem. Partindo dos conceitos que nutriram a Reforma Sanitária e a construção dos princípios do SUS, o caminho metodológico empreendido utilizou entrevistas com os coordenadores do programa. A análise permitiu reconhecer que a política e as práticas da gestão municipal estavam comprometidas com o SUS; priorizou-se a qualificação dos trabalhadores da saúde que não haviam tido oportunidade; adotou-se uma pedagogia voltada à "transformação" dos agentes; os coordenadores motivaramse a desenvolver a qualificação, reconhecendo o processo como uma ferramenta de implementação do SUS; os coordenadores do nível regional apreenderam a dimensão técnica do processo, isolada da política.

DESCRITORES: SUS (BR), educação em enfermagem, recursos humanos de enfermagem, política de saúde, capacitação em serviço

\section{THE POLITICAL DIMENSION OF AUXILIARY PERSONNEL EDUCATION: NURSING AND THE UNIFIED HEALTH SYSTEM (SUS)}

The change in the administration of the municipality of São Paulo in 1989 innovated the management of health services aiming at implementing the Unified Health System (SUS) and using as a fundamental strategy the workers' qualification. Thus, the purpose of this work was to analyze the incorporation of the SUS principles in the programs for the capacitation of nursing auxiliary personnel. Based on the concepts that nurtured the Sanitary Reform and the construction of SUS principles, the methodological framework comprehended interviews with the coordinators of the program. Data analysis enabled authors to recognize that the municipal management policies and practices were committed to SUS guidelines, giving priority to the qualification of health workers who did not have this opportunity and adopting a pedagogy directed to the agents' transformation. The coordinators were motivated to develop the qualification program, recognizing this process as a tool for the SUS implementation. In addition, the coordinators at the regional level understood the technical dimension of this process, isolated from its political dimension.

\section{LA DIMENSIÓN POLÍtICA DEL PROCESO DE FORMACIÓN DEL PERSONAL AUXILIAR: LA ENFERMERIA RUMBO AL SUS (SISTEMA ÚNICO DE SALUD)}

El cambio de administración en el Municipio de São Paulo, en 1989, innovó la gestión de los servicios de salud, buscando la implementación del Sistema Único de Salud, (SUS) teniendo como estrategia fundamental la cualificación de los trabajadores. Este artículo analizó la incorporación de los principios del SUS en la formación de auxiliares de enfermería. Se realizaron entrevistas con los coordinadores del programa y su análisis permitió reconocer que: las políticas y prácticas de la gestión municipal estaban comprometidas con el SUS; se priorizó la cualificación de los trabajadores de la salud que no habían tenido oportunidad; fue adoptada una pedagogía orientada hacia la "transformación" de los agentes; los coordinadores se motivaron para desarrollar esa cualificación, reconociendo el proceso como una herramienta para implementar el SUS; los coordinadores regionales aprehendieron la dimensión técnica del proceso, aislada de la política.

DESCRIPTORES: SUS (BR), educación en enfermería, recursos humanos, política de salud, capacitación en servicio

\footnotetext{
${ }^{1}$ Este trabalho foi aceito para ser apresentado no VII Congresso Paulista de Saúde Pública - Saúde e Democracia, a ser realizado no período de 29 de setembro a 3 de outubro de 2001, em Santos-SP; ${ }^{2}$ Enfermeira do Núcleo de Formação do Centro de Formação dos Trabalhadores da Saúde - Secretaria Municipal de Saúde da Prefeitura do Município de São Paulo, Mestre em Saúde Pública pela Faculdade de Saúde Pública, da Universidade de São Paulo, e-mail: ceforsms@yahoo.com.br; ${ }^{3}$ Enfermeira, Mestre em Saúde Pública, Doutor em Educação, Professor da Escola de Enfermagem da Universidade de São Paulo, e-mail: cassiaso@usp.br
} 


\section{INTRODUÇÃO}

Esta pesquisa trata da implementação dos cursos de formação de auxiliar de enfermagem no município de São Paulo, durante a gestão do Partido dos Trabalhadores (PT), de 1989 a 1992. O objeto constitui-se mais propriamente nas relações estabelecidas entre a prática adotada para a qualificação do trabalhador de saúde em serviço - o Projeto Larga Escala (PLE) - e a orientação da política pública de saúde da administração municipal, discutindo a dimensão política desse processo, para além dos aspectos técnicos e pedagógicos que, mais freqüentemente, vêm sendo objeto de preocupação da área de enfermagem.

A problemática da inadequação da formação dos trabalhadores de saúde frente às necessidades dos serviços, tanto quantitativa quanto qualitativamente, vem sendo referida em documentos oficiais há pelo menos três décadas ${ }^{(1)}$. Poucas foram, no entanto, as experiências de impacto implementadas no país ao longo desse período $^{(2)}$.

Os analistas da área concordam com que a década de 80 representou um período de intensa absorção pelas instituições de saúde de mão-de-obra despreparada e de baixo custo, constituída pelas atendentes, a fim de viabilizar a expansão da rede de serviços no país ${ }^{(1,3-5)}$. 0 contingente de trabalhadores sem qualificação específica para o trabalho significava $70 \%$ do pessoal empregado no setor, sendo que $50 \%$ desenvolvia ações na área de enfermagem ${ }^{(3-5)}$.

A consolidação das diretrizes e dos princípios do Sistema Único de Saúde (SUS), postulado, ainda que parcialmente, a partir do ideário do movimento da Reforma Sanitária, acabou por demandar dos serviços de saúde, centros formadores, escolas técnicas e universidades, uma nova lógica na formulação de políticas e no desenvolvimento de práticas de formação do trabalhador. Estabeleceu-se, desde a I Conferência Nacional de Recursos Humanos, em 1986, um "consenso técnico e político", entre os principais pesquisadores da área, quanto à necessidade de ampliar o espaço de influência desse novo modo de organização dos serviços de saúde sobre o processo educacional dos agentes dessa prática ${ }^{(2)}$.

A tarefa demandava a construção de um novo paradigma também na arena educacional, requerendo a participação ativa do trabalhador na construção do SUS ${ }^{(4)}$, recuperando e valorizando a dimensão criativa do agente em formação e instaurando um novo patamar de articulação ensino-serviço que aproximasse os dois mundos ${ }^{(6-8)}$.

A administração pública do município de São Paulo, do período de 1989 a 1992, assumiu o governo com o compromisso de inverter as prioridades do investimento público e governar para "atender prioritariamente as necessidades e os direitos sociais da população"(9). Dessa forma, as principais propostas da área da saúde estavam "centradas na participação popular, na descentralização, e na democratização dos serviços de saúde, tendo por objetivo a implementação do SUS na cidade de São Paulo"(10).

O projeto de reorganização dos serviços de saúde exigiu, estrategicamente, a adoção de uma política comprometida com a melhoria da inserção dos trabalhadores nos serviços de saúde. Assentando-se nos princípios da valorização, profissionalização e participação dos funcionários, tal decisão buscava produzir mudanças na mentalidade e nas ações, mediante a qualificação e a incorporação de novos agentes. Propunha-se, dessa forma, a recuperar uma "postura de respeito e dignidade ao trabalho e aos trabalhadores" ${ }^{(11)}$.

A prioridade foi a profissionalização dos trabalhadores de nível médio e elementar, que em 1989, correspondiam a $50 \%$ do total de servidores existentes na Secretaria Municipal de Saúde (SMS) ${ }^{(11)}$ : adultos com baixa escolaridade, sem qualificação específica para o trabalho e que, normalmente, não constituíam o alvo de treinamentos ou atualizações ${ }^{(2)}$. Dessa forma, a SMS, por meio do Centro de Formação dos Trabalhadores da Saúde (CEFOR), implementou cursos de formação profissional mediante o desenvolvimento do PLE, junto às Administrações Regionais de Saúde (ARSs). A opção por um projeto de formação como esse - estruturado a partir da prática profissional -, representava a efetiva experiência dos serviços de saúde em realizar a capacitação do pessoal auxiliar, adequando-se às características do atendente ${ }^{(2)}$.

O PLE foi resultado de um acordo interministerial, envolvendo Saúde, Educação e Cultura, Previdência e Assistência Social e a Organização Panamericana da Saúde, em 1982, a fim de qualificar o pessoal de nível médio e elementar empregado nos serviços básicos de saúde. O projeto adotava o trabalho como princípio pedagógico, tendo como ponto de referência a realidade da prática dos alunos, utilizava como corpo docente os próprios profissionais da rede de serviço, garantindo aos egressos a identidade profissional por meio do reconhecimento pelo sistema formal de ensino ${ }^{(2)}$. 
Por seu turno, a nova Lei do Exercício Profissional de Enfermagem, em vigor a partir de 1986, determinava o prazo de dez anos para a profissionalização das atendentes, reconhecendo, como profissionais da equipe de enfermagem, apenas três categorias: Auxiliar, Técnica e Enfermeira.

Para a gestão municipal em questão, a adoção desse projeto de formação como um instrumento para "provocar mudanças no comportamento dos trabalhadores, que thes permitissem refletir sobre a organização dos serviços, a partir do reconhecimento da função social do trabalho em saúde", deveria atender aos princípios finalísticos e às diretrizes do SUS ${ }^{(2)}$.

Partindo do pressuposto de que o PLE representou para essa gestão municipal uma estratégia de implementação do projeto político do setor e do ideário da reforma sanitária, este trabalho objetivou analisar a incorporação dos princípios e das diretrizes políticas do SUS - descentralização, universalidade, integralidade, eqüidade e participação popular - aos conteúdos e estratégias dos cursos de formação do Auxiliar de Enfermagem do PLE, no período de 1989 a 1992. Configurou-se como uma possibilidade de explicitar a relação ocorrida entre o espaço político das ações desenvolvidas pela administração municipal e a compreensão dos trabalhadores da saúde sobre as propostas educativas referenciadas naquelas ações ${ }^{(2)}$.

\section{PROCEDIMENTOS METODOLÓGICOS}

Este estudo, de natureza qualitativa, constitui-se numa pesquisa estratégica que utilizou como instrumento entrevistas semi-estruturadas, realizadas no $2^{\circ}$ semestre de 1999, junto a profissionais que participaram do desenvolvimento do projeto de formação na referida administração municipal. Dois tipos de roteiros foram elaborados e testados junto a profissionais que não participaram da amostra. Foram entrevistados sujeitos dos níveis Central (2) - direção do CEFOR - e Regional (5) Administrações Regionais de Saúde/Distritos Sanitários - da SMS.

Para o nível central, serviu de base um roteiro de entrevista bastante abrangente, uma vez que, nesse nível, buscavam-se informações sobre o contexto e a direção técnica e política mais geral que orientava a SMS e o planejamento das ações do CEFOR, quanto à formação de recursos humanos, sendo exemplarmente selecionados dois trabalhadores considerados elementos-chave na gestão do CEFOR, uma vez que participaram do planejamento da implantação do PLE.

Para o nível regional, o roteiro limitou-se ao objeto mais específico da pesquisa, procurando esclarecer a contribuição do PLE para a implementação dos princípios e diretrizes do SUS, sendo igualmente selecionados exemplarmente três profissionais que participaram diretamente na função de instrutor-coordenador, ministrando o PLE, e dois que atuaram na função de gerentes, coordenando regionalmente a implantação do PLE - um da equipe de Recursos Humanos e um do Serviço de Enfermagem.

Dado o fato de que a atividade de formação de recursos humanos fora descentralizada nas ARSs e, considerando que o desenvolvimento dessas práticas fora diferenciado em cada uma das regiões, ajustando-se às particularidades internas nos diferentes Distritos de Saúde (DSs), o processo de escolha dos sujeitos de pesquisa tentou acompanhar essa diversificação, selecionando-se entrevistados das diferentes regiões do município, escolhidos intencionalmente pelo seu reconhecido esforço, na implementação do PLE.

O projeto de pesquisa foi aprovado pelo Comitê de Ética da Faculdade de Saúde Pública da Universidade de São Paulo, tendo os participantes assinado um Consentimento Informado.

As entrevistas foram gravadas e transcritas pela pesquisadora, sendo posteriormente categorizadas por meio de análise temática ${ }^{(12)}$. Tratou-se de reconhecer, entre os dados empíricos, os conteúdos que expressassem o conjunto de idéias remetido a alguns dos princípios e diretrizes do SUS, utilizados como temas: descentralização, universalidade, integralidade, eqüidade e participação popular. Os fundamentos teóricos utilizados para construir esses temas enquanto categorias tiveram respaldo na Constituição Brasileira, na Lei Orgânica da Saúde (LOS) e na contribuição de diversos estudiosos dos temas pré-selecionados. Assim, na análise dos dados, buscou-se estabelecer relações entre os referenciais teóricos propostos pelo projeto político da Reforma Sanitária Brasileira e os resultados obtidos nas entrevistas.

\section{RESULTADOS}

Descentralização e participação popular constituíram-se nas diretrizes mais enfatizadas pelos 
entrevistados. O compromisso da administração com a implantação do SUS no município de São Paulo determinou, para os trabalhadores engajados no nível central, a tarefa de reorganizar os equipamentos de saúde, além de efetivar profundas mudanças nas práticas desenvolvidas.

Na perspectiva mais geral que se tinha de governo, a idéia era, em primeiro lugar, de se fazer a municipalização dos serviços (....) assumir as unidades básicas da Secretaria de Estado da Saúde (....) criar um processo de regionalização da cidade (....) Então, a idéia era descentralizar a cidade de São Paulo e não ser só a saúde que se descentralizaria, mas todas as demais Secretarias deveriam se dividir em Administrações Regionais e criar sub-prefeituras ou prefeituras regionais ( $D 2)$.

A reorganização da SMS, com a criação das ARSs e dos DSs, foi majoritariamente entendida pelas entrevistadas engajadas no nível regional, como uma prática oriunda da diretriz da descentralização da gestão de saúde, tanto no seu sentido político, quanto administrativo e assistencial. A municipalização dos serviços foi mesmo tratada por uma das entrevistadas, como sendo o principal desafio político daquela gestão, tendo chegado a representar, na opinião de uma outra profissional, o cumprimento do compromisso com a implantação do SUS no município de São Paulo.

Eu acho que, enquanto política de saúde, foi num momento em que a Administração tinha um compromisso com a implantação do Sistema Único de Saúde, e um compromisso muito claro de estar fortalecendo o Sistema Único de Saúde, de estar fazendo com que o que estava na lei, o que estava sendo proposto para o SUS, que ele se tornasse de fato, que ele de fato acontecesse (D 6).

Assim, o projeto de formação se configurou como uma das propostas do processo de descentralização, uma proposta comprometida com a capacitação das equipes de trabalho, a fim de viabilizar uma nova gestão em saúde.

A gestão abriu caminho para a incorporação de novas práticas em saúde, particularmente voltadas para o novo objeto da saúde coletiva - os problemas e necessidades de saúde no nível local - e para a nova finalidade do trabalho em saúde - o enfrentamento da situação de saúde no nível local.

As formas como os profissionais envolvidos com a preparação dos trabalhadores perceberam e desenvolveram essas mudanças, foram significativamente diversas. Em todas as regiões de saúde, com particularidades, as novas práticas foram introduzidas e incorporadas aos conteúdos dos cursos de formação. No entanto, a minoria das entrevistadas as reconheceu como propostas de efetivação da descentralização.

Assim, o entendimento sobre Recursos Humanos em Saúde também era novo, 'estratégico para fazer funcionar o serviço de saúde' e coerente com o projeto político que se propunha implantar, ou seja, o trabalhador deveria deixa[r] de ser visto como mais um 'insumo' e passa[r] a ser visto como um 'sujeito' com necessidades, desejos, direitos, valores, formas de pensar etc ( $D$ 1).

A compreensão a respeito do que seria caminhar no sentido da diretriz da participação popular foi mais amplo do aquele expresso na Lei Complementar 8.142, de 1990. Os trabalhadores de âmbito central lembraram que, de acordo com os pressupostos da Reforma Sanitária, a implementação do SUS pressupunha a participação da sociedade civil, por intermédio de suas organizações representativas (ou diretamente), no atendimento de suas demandas.

[Considerando os movimentos sociais existentes no contexto político pré-eleições e, particularmente, o movimento de saúde], o que era mais claro para esses movimentos era justamente, a perspectiva do controle público, do controle social dos equipamentos por meio da construção de um Conselho Gestor nas Unidades de Saúde (D 2).

Mas deram importância também ao modo como a profissionalização dos trabalhadores foi desenvolvida, a partir do ambiente de trabalho, por meio de uma pedagogia facilitadora que promovesse o desenvolvimento de sujeitos críticos, que tivessem por referência as realidades locais de saúde e reconhecessem a importância da participação (própria e dos demais usuários dos serviços) na defesa dos seus interesses.

A idéia era que os usuários tivessem ampla maioria e que a unidade dos trabalhadores de saúde com os usuários enquanto unidade potencialmente estratégica, pudesse ser uma ampla maioria, que forçasse as políticas públicas num certo rumo (D 2).

Todas as entrevistadas engajadas no trabalho de formação de âmbito regional entenderam a participação num sentido mais amplo do que a participação popular, fazendo referências à possibilidade de que o trabalhador pudesse participar efetivamente de todos os processos de trabalho em saúde.

Reconheceu-se que o contexto político propiciava liberdade/possibilidade de participação na condução do trabalho e era favorável e estimulante à participação dos profissionais de uma maneira geral - professor ou aluno-, assim como da população usuária dos serviços. 
(....) e o que mais me impressionou assim, me deu mais vontade de trabalhar, abraçar a causa mesmo, foi a liberdade que a gente tinha de expressão, de trabalho, (....) com respeito...foi uma abertura maior... a própria população, uma participação maior da população, da comunidade (...) a nossa grande conquista é o envolvimento da população, o envolvimento dos nossos alunos, dos nossos funcionários, a equipe que a gente estava trabalhando, eu acho que foi a melhor coisa na época...(D 3).

$\mathrm{Na}$ verdade, as estratégias metodológicas da capacitação foram valorizadas pelas entrevistadas como instigantes do comportamento participativo, uma vez que propiciavam discussão, possibilitando apreensão das condições de vida e trabalho da população usuária e, portanto, melhorando a compreensão a respeito do conjunto de necessidades de saúde da população e da importância da participação na busca da resolução dos problemas. Assim, a própria participação dos alunos, favorecida por essas estratégias, concorreria para sedimentar uma visão crítica e ampliada da realidade, o compromisso com o trabalho e o respeito ao usuário.

Duas entrevistadas compartilharam o entendimento que não somente as estratégias metodológicas, mas também a eleição de alguns conteúdos possibilitavam aos alunos conhecer e, muitas vezes, identificar-se com a realidade de vida e saúde da população e dessa forma desenvolver uma postura solidária ao direito à saúde da população.

Durante aquele período, nós tínhamos liberdade para desenvolver um trabalho com tranqüilidade, (....) fazendo com que o nosso pessoal atendente, o nosso público alvo que eram os atendentes, eles passaram a ter uma outra visão, da própria comunidade, da importância da saúde pública, (....) porque eles faziam muito trabalho de campo, de sistematizar, de problematizar, as dramatizações... (D 3).

(....) Então a proposta é que ele se relacionasse com a comunidade como ele participante daquele meio, aquela comunidade era dele também e, por meio disso, ele tomando essa consciência, ele passou a tratar melhor o usuário, a se sentir daquele contexto porque o comum, era ele não se sentir dentro daquele contexto... ( $D$ 5).

No que se refere à integralidade, foi possível perceber que os depoimentos do nível central mostraram preocupação com a implantação de programas de atenção à saúde sustentados por uma lógica diversa.

Partia-se de um princípio que era de trabalhar com a equipe multidisciplinar e buscar uma prática que não fosse centrada no médico, na assistência médica (D 1).
As mudanças implementadas tinham como meta alterar padrões de serviços estabelecidos, ao longo dos anos, e caracterizados pelo atendimento médico hospitalar de urgência e por unidades de assistência médica ambulatorial.

Então você tinha um atendimento médico individual de caráter hospitalar e de urgência, que era a grande marca, você não tinha Unidades Básicas de Saúde na rede de serviços exceto, algumas unidades na área da saúde escolar (....) Eram muito poucas as estruturas ambulatórias e todas elas eram especializadas e verticais (....) cada uma tinha um mando próprio e à parte, e não tinham muita conversa entre si (...) Existia um fosso entre os hospitais e a rede básica. Era isso que precisava mexer de algum jeito, de unir as coisas... (D 2).

Para atingir a integralidade, as ações em saúde deveriam.

Colocar a assistência médica individual não como fim, mas como parte, como um instrumento de uma política mais geral, mais orientada para a saúde coletiva (....) onde as ações curativas individuais tivessem um caráter de certa forma subordinado às características epidemiológicas, das determinações sociais do processo saúde-doença de cada bairro, de cada região... (D 2).

$A$ análise dos dados permitiu constatar que houve compromisso dos instrutores-coordenadores com o ensino dos conceitos e das práticas que se referem à integralidade das ações, inclusive através da decisão de montar turmas mistas de alunos, reunindo aqueles procedentes das Unidades Básicas com aqueles de Hospitais. No desenvolvimento dos cursos, buscava-se valorizar os conhecimentos específicos dos diferentes profissionais da equipe de saúde, na perspectiva da complementaridade do trabalho multidisciplinar. Além disso, as estratégias metodológicas utilizadas propiciavam aos alunos compreender a teoria da determinação do processo saúde-doença, a partir da concretude dos determinantes sociais, ampliando-Ihes a visão e as possibilidades de atuação profissional.

Os princípios da universalidade e da eqüidade foram referidos pelos entrevistados do nível central num sentido amplo, sustentado pelos interesses políticos mais gerais da Reforma Sanitária, de viabilizar a idéia do poder local, poder público... atender a todos com igualdade, aumentar e viabilizar o acesso à saúde da população, numa perspectiva de universalizar o atendimento da cidade e fazer da saúde um instrumento de justiça social, de redistribuição, de poder, de qualidade de vida (D2).

Além do enfoque histórico e político, os dados 
empíricos do estudo permitiram constatar que os princípios da eqüidade e da universalidade foram apreendidos de forma particular na área de Recursos Humanos. A eqüidade foi reconhecida não somente como um princípio de promoção da justiça no que se refere à atenção à saúde da população, mas também como um princípio que buscasse promover justiça, criando oportunidades de qualificação profissional, para os trabalhadores que, tradicionalmente, não eram os escolhidos para o desenvolvimento de treinamentos e atualizações, rompendo, assim, com o processo de desigualdade gerada no interior das organizações dos serviços de saúde. Por outro lado, a extensão dessas oportunidades a todos os trabalhadores, de forma irrestrita, significaria, também, o respeito à universalidade.

Também no nível regional, ocorreu a apropriação dos conceitos que ampararam os princípios da eqüidade e da universalidade de uma forma particular, no interior da política de $\mathrm{RH}$ e diversa daquela apresentada na Constituição Federal. O PLE foi visto como uma estratégia de promoção de justiça para com os trabalhadores que nunca haviam tido oportunidade de qualificação. $\mathrm{Na}$ mesma direção, a universalidade foi compreendida no sentido de garantir que essa política fosse estendida a todos os trabalhadores.

(....) quando vinha papel de curso no nosso posto ou era pra médico, ou pra assistente social, ou psicólogo, nunca vinha nada pro atendente, "a gente sempre foi colocada de lado", nunca teve curso de reciclagem nada! (D4).

A análise dos dados mostrou, também, diferentes entendimentos a respeito da vinculação do desenvolvimento do projeto de formação ao princípio da eqüidade. Uma das entrevistadas entendeu que a melhoria da qualidade do trabalho seria atingida por meio da formação, devido à coexistência no curso de algumas condições facilitadoras: a sua realização dentro do próprio horário de trabalho do aluno; a utilização das próprias unidades de serviço como locais de prática, evitando-se, dessa forma, o deslocamento do aluno para locais diferentes; o reconhecimento das suas experiências como ponto de partida para as suas reflexões, entre outras.

A formação do trabalhador dentro do seu próprio horário de trabalho, sem ter que fazê-lo deslocar de um lugar para outro, e mesmo pela falta de condições de pagar, porque a grande maioria daqueles profissionais (...) não tinha recursos (...), com o surgimento do projeto o que aconteceu, ele teve a oportunidade da formação, dentro do horário de trabalho e, conseqüentemente, melhorar as condições para ele próprio ter uma visão real (....) utilizando o seu próprio local para a parte prática, principalmente, em cima do conhecimento que ele já tinha (....) então isso avançou, progredia muito o nosso desenvolvimento (....) (D3).

Numa outra entrevista, um relato testemunhou um certo estranhamento que o desenvolvimento do curso provocava no grupo de alunas. As alunas acabavam cobrando o ensino das técnicas básicas da enfermagem, apreensivas que estavam com o uso do tempo do curso para discussão.

De outro lado, paradoxalmente, o grupo aplaudia as experiências que lhe permitiam um espaço para reconhecer as diferenças estabelecidas nas relações de trabalho, valorizar as suas próprias experiências, trazendoas para as salas de aula, exercer sua opinião e participar. Reconhecia-se que, concretamente, estava abrindo-se a possibilidade de se afastar do papel alienante do trabalhador que simplesmente executa tarefas para assumir o papel do trabalhador que compreende essas tarefas como parte dos processos de trabalho que concorrem para a produção de serviços de saúde, com vistas ao objeto do trabalho em saúde.

Houve somente uma citação que relacionou o PLE ao princípio da universalidade. A entrevistada, além de avaliar a efetividade do PLE, reconhecer o caráter democrático do processo de formação das turmas e o espírito de justiça embutido na prioridade dada aos trabalhadores que não tinham tido qualificação até então, deu destaque à intenção universalizadora da proposta de formação que visava dar oportunidade a todas, gradualmente.

(....) Mas acontece que a escolha das pessoas tinha sido um processo conjunto, bastante democrático, tinha sido decisão delas de se dar oportunidade pra quem não tinha tido nenhuma até então... E o nosso entendimento, que nós íamos começar com essas pessoas, que elas próprias escolheram para formar, e depois, em outro momento, formaríamos outras, até chegar a formar todo mundo. Todo mundo ia ter a mesma oportunidade, em outro momento... (D4).

\section{DISCUSSÃO E CONSIDERAÇÕES FINAIS}

A decisão política de âmbito central de formar as auxiliares de enfermagem configurou-se como um componente importante de uma política de viabilização do novo modelo de atenção à saúde, implantado no 
município de São Paulo, no período de 1989 a 1992. Havia clareza da necessidade de preparar as pessoas que iriam reconstruir a prática, tendo por norte os princípios que orientavam o projeto político do setor e por referência à reforma processual das condições sanitárias do país, desenvolvendo a dimensão política do projeto de formação de trabalhadores de nível médio ${ }^{(13)}$.

Assim, implementar o PLE de uma maneira descentralizada significou capacitar as atendentes de enfermagem a desenvolver a prestação de serviços de saúde dirigidos para uma determinada realidade local, constituída pela situação de saúde de uma dada população e pela reordenação dos serviços de saúde que buscavam mudanças, incorporando profissionais e ações na perspectiva da atenção integral, promovida por um trabalho de equipe. Sobre isso, outra pesquisa ${ }^{(14)}$ reitera o entendimento quanto à intenção da SMS em promover o trabalho interdisciplinar. Para esse autor, "o trabalho coletivo, em equipe, fortalece a tese da integração, uma vez que são reforçadas as possibilidades de integração entre os diferentes saberes no vasto campo da saúde coletiva".

Para além dos foros centrais de decisão das práticas estratégicas para as políticas adotadas, o PLE foi incorporado como sendo de responsabilidade do nível local. Além do PLE, outras reformulações de caráter técnico-gerenciais foram implementadas pelas ARSs e incorporadas aos conteúdos dos cursos de formação, o que, porém, não foi reconhecido no conjunto das propostas de efetivação da descentralização.

O entendimento da descentralização vinha de encontro ao conceito apresentado por outro autor ${ }^{(15)}$ "um processo de natureza político-comportamental, pelo qual ocorrem redefinições entre centro e periferia, através da transferência significativa de poder, e cujo significado seria dar competência aos pontos mais afastados do centro, ampliando suas áreas de atuação através de iniciativas próprias locais".

A perspectiva da participação, ora relativa à participação dos trabalhadores nas decisões do processo de trabalho, ora relacionada à participação da população nas decisões concernentes à situação de saúde e de atenção à saúde, foi integrada tanto por aqueles que eram responsáveis por pensar as políticas de qualificação dos trabalhadores, quanto por aqueles que, no dia a dia, eram responsáveis pelo desenvolvimento das práticas associadas a essas políticas.
O princípio da integralidade também foi fortemente relacionado aos conteúdos dos cursos. Houve, de fato, um entendimento de que a associação de determinados conhecimentos à utilização de algumas estratégias metodológicas propiciariam uma visão ampliada da atenção à saúde e adequada às mudanças projetadas e esperadas.

Um dos princípios desse projeto de formação foi o de considerar a prática profissional como a essência para a aprendizagem. Portanto, enfrentou o desafio de reconstruir o conhecimento - até então baseado apenas em técnicas assistenciais isoladas do contexto de trabalho no qual as atendentes se inseriam - para adotar um conhecimento baseado tanto na realidade das condições de vida da população quanto nas reformulações dos processos de trabalho. Tal processo demandou das alunas uma postura de discussão, observação, discernimento, ponderação, identificação e, por fim, participação.

Para os entrevistados que estavam em sala de aula, não houve uma associação clara entre a proposta política do SUS - centrada nos seus princípios e diretrizes - e o processo educativo do PLE, ou seja, participar da proposta educativa, das decisões de uma comunidade para resolver problemas de saúde, ou ainda, dos projetos novos para entender as mudanças, não se configuraram como interesses políticos daquela gestão no sentido de operacionalizar a sua orientação teórica.

O princípio da eqüidade foi visto, com destaque, sob a ótica da política de RH e não sob a ótica da distribuição de recursos de saúde. Era certo que a SMS não investia na capacitação profissional voltada para as categorias de nível médio e auxiliar. No geral, as atividades de formação, desvinculadas da atuação em serviço, eram dirigidas para os trabalhadores de nível universitário"(16) Assim, ao voltar o olhar para as atendentes, promovia-se a eqüidade.

Nossos dados revelaram-se coerentes com a discussão apresentada por outro autor ${ }^{(14)}$ que identificou, na opinião dos dirigentes entrevistados, o "reconhecimento de que a Secretaria promoveu a eqüidade ao organizar todo o processo de desenvolvimento dos recursos humanos e na incorporação de novas tecnologias que possibilitaram a resolução de problemas de saúde dos grupos mais desfavorecidos".

Os distintos processos de descentralização da gestão de saúde, viabilizando a criação dos DS(s), não pareceram ter se constituído um contexto político facilitador de uma compreensão ampliada das propostas políticas do período estudado. 
Ainda que a decisão de priorizar os trabalhadores de nível médio e elementar, no processo de qualificação profissional, tenha se constituído numa decisão política comprometida com a promoção da justiça, as capacitações pedagógicas e técnicas, realizadas junto aos profissionais do nível universitário, não foram suficientes para proporcionar-Ihes a compreensão da dimensão política do processo educativo.

\section{REFERÊNCIAS BIBLIOGRÁFICAS}

1. Fundação Oswaldo Cruz. Escola Nacional de Saúde Pública. A política de recursos humanos em saúde no Brasil nos anos recentes. In: Médici AC, organizador. Recursos humanos em saúde. Rio de Janeiro: PEC/ENSP/ABRASCO; 1987. p. 24-36. (Textos de Apoio Planejamento, 1).

2. Almeida $A H$. A incorporação dos princípios e das diretrizes do Sistema Único de Saúde aos cursos de formação do auxiliar de enfermagem - projeto larga escala - 1989 - 1992. [dissertação]. São Paulo (SP): Faculdade de Saúde Pública/ USP; 2000

3. Nogueira RP. Dinâmica do mercado de trabalho em saúde no Brasil 1970-1983. Brasília (DF): OPAS - Acordo MS/MPAS/ MEC/MCT; 1986.

4. Vieira TCV, Scucato R. Formação de pessoal auxiliar para enfermagem nos serviços de saúde: Brasil. Educ Med Salud 1988; 22:35-46.

5. Santos I, Souza AA. Formação de pessoal de nível médio pelas instituições de saúde: projeto larga escala, uma experiência em construção. Saúde Deb 1989; 61-4.

6. Santana JP. Formulação de políticas de RH nos serviços de saúde. Saúde Deb 1990; (28):66-71.

7. Oliveira M Junior. Administração de recursos humanos um obstáculo à municipalização dos serviços de saúde? Saúde Debate 1990; (28):41-5.

8. Paim JS. Recursos humanos em saúde no Brasil: problemas crônicos e desafios agudos. São Paulo: Faculdade de Saúde Pública/USP; 1994.

9. Jacobi P. Políticas públicas e alternativas de inovação da gestão municipal: o complexo caso da cidade de São Paulo. São Paulo Perspect 1991; 5(2):31-7.

10. Cohn A, Elias P, Jacobi P. Participação popular e gestão de serviços de saúde: um olhar sobre a experiência do município de São Paulo. Saúde Deb 1993; (38):90-3.

11. Secretaria Municipal da Saúde. (SP). Proposta de formação de pessoal de níveis elementares e médio na Secretaria Municipal de Saúde. São Paulo: SMS; 1989.

12. Bardin L. Análise de conteúdo. Lisboa: Edições 70; 1977. 13. Torres MNFB. Qualificação e trabalho em saúde o desafio de "ir além" na formação dos "trabalhadores" de nível médio. [dissertação]. Rio de Janeiro (RJ): Faculdade de Educação/ UFRJ; 1994.

14. Telesi E Junior. Reflexões sobre a política de saúde do município de São Paulo (1989-1992). [tese]. São Paulo (SP): Faculdade de Saúde Pública/USP; 1998.

15. Sampaio GM, Parente LI, Wohland M. Uma experiência de descentralização em administração pública. Cad FUNDAP 1987; (13):23-6.

16. Mattos ML. Recursos humanos e o novo trabalhador em saúde: a experiência da Secretaria Municipal de Saúde na cidade de São Paulo durante a gestão do partido dos trabalhadores (1989-1992). [dissertação]. Campinas (SP): Faculdade de Ciências Médicas/UNICAMP;1996. 